\title{
Thermal capacitors made from Miscibility Gap Alloys (MGAs)
}

\author{
T. Fiedler, A. J. Rawson, H. Sugo \& E. Kisi \\ School of Engineering, The University of Newcastle, Australia
}

\begin{abstract}
The current paper addresses the thermal characterisation of Miscibility Gap Alloys (MGAs). These novel materials combine two immiscible metallic phases with different melting temperatures. The fusible phase (i.e. the phase with a lower melting temperature) acts as a phase change material that stores latent heat (in addition to sensible heat) thus optimising energy storage capacity. The second phase forms an enclosure and prevents the leakage of liquid material. Due to the high inherent thermal conductivity of metals, MGAs exhibit excellent thermal conduction in comparison to traditional phase change materials such as hydrate salts or paraffin. The combination of high energy storage and fast heat transfer makes MGA uniquely suited for use as thermal capacitors in applications like space heating, concentrated power generation or temperature stabilisation of sensitive equipment. The current paper determines the thermal properties of MGAs using Lattice Monte Carlo analysis combined with micro-computed tomography imaging.
\end{abstract}

Keywords: thermal storage, Miscibility Gap Alloy, micro-computed tomography, Lattice Monte Carlo.

\section{Introduction}

Miscibility Gap Alloys (MGAs) combine high thermal diffusivity with an excellent heat storage capacity. Due to these unique properties MGAs are excellent materials for thermal capacitors and enable the rapid storage and discharge of thermal energy. An important area of application is concentrated solar power generation where thermal capacitors allow the compensation of power intermittency (i.e. due to cloud coverage) and the extension of power generation towards peak consumption after sunset. 
MGAs are composites containing two or more metallic phases. A fusible phase is completely enclosed by a second one with a significantly higher melting temperature. In the current study, iron-copper and aluminium-tin systems are considered where the second phase is the fusible one. A high thermal diffusivity of the composite is ensured due to the high inherent thermal conductivity of metals. Energy storage capacity is strongly increased by latent energy storage during the phase transition of the fusible phase. The selection of the material system allows the phase transition temperature to be adapted to the optimum operation temperature of the thermal capacitor.

There exist a large number of materials suggested as potential Thermal Energy Storage (TES) media in the literature. Numerous review papers exist in a number of journals listing and categorising thermal storage materials on characteristics and temperature range [1-8]. The state of the art in thermal storage materials may be categorised as sensible or phase change systems.

Sensible TES systems do not utilise a phase change and thus rely completely on the heat capacity and temperature range of the storage material. Sensible storage material general have high heat capacity $(>1000 \mathrm{~J} / \mathrm{kg} . \mathrm{K})$ and are cheaper than phase change systems.

Solid sensible media like cast iron, concrete, graphite, rocks, and sand are commonly encountered in literature [4, 9-11]. These materials are generally cheap with poor energy density $(<0.1 \mathrm{kWh} / \mathrm{L})$ and low thermal conductivity $(<5 \mathrm{~W} / \mathrm{m} . \mathrm{K})$. Cast Iron was identified as having a better energy density than water by Hasnain, though the much higher cost was also mentioned [10]. Laing investigated N4 concrete as a potential solution [4]. Concrete based storage was found to suffer from low thermal conductivity $(<1.5 \mathrm{~W} / \mathrm{m} . \mathrm{K})$ [12] and had some issues with elimination of water. Graphite shows some potential due to its high thermal conductivity (up to $200 \mathrm{~W} / \mathrm{m} . \mathrm{K}$ ) and moderate heat capacity ( 700 J/kg.K) [11]. Beds of rocks and sand were also reviewed by Hasnain with favourable results for moderate temperatures [10].

Liquid sensible storage media are almost invariably molten salts. A huge number of molten salts have been identified as potential storage materials with exceptional heat capacities ( $>1000 \mathrm{~J} / \mathrm{kg} . \mathrm{K})$ [13]. The most prevalent and investigated molten salts are Potassium and Sodium Nitrates. Unfortunately all molten salts discussed in the literature suffer from extremely low thermal conductivity $(<1 \mathrm{~W} / \mathrm{m} . \mathrm{K})$ and most are very reactive with steel [10]. Most salts are also likely to decompose to potentially hazardous chemicals at moderate temperatures [10]. Thermal oils have also been used as liquid sensible storage materials. These oils are restricted in upper operating temperature $\left(>300^{\circ} \mathrm{C}\right)$ due to chemical decomposition and also suffer from very low thermal conductivity $(<1 \mathrm{~W} / \mathrm{m} . \mathrm{K})$ [8]. The need to pump these molten storage materials adds a parasitic energy loss to the entire plant $(\sim 10 \%)$ reducing overall efficiency.

Phase change TES systems have higher energy density and may operate over smaller temperature ranges than sensible heat systems. Researchers have identified many salts and salt eutectics, paraffin waxes and other materials that undergo phase changes at useful temperatures. These materials generally have low conductivity $(<1 \mathrm{~W} / \mathrm{m} . \mathrm{K})$ and higher implementation costs. Their energy density 
$(0.2-0.7 \mathrm{kWh} / \mathrm{L})$ is much higher than sensible storage media, thus less material is required to achieve a required energy capacity. Limited work has involved the use of metals as phase change materials.

Salts and eutectics of salts are very widely documented in the literature $[1,5,8,13]$. Operating temperatures range from $10^{\circ} \mathrm{C}$ up to near $1700^{\circ} \mathrm{C}[13]$ with some rather exotic salts suggested. The most commonly investigated salts for phase change storage include fluorides, chlorides, hydroxides, nitrates and carbonates based on cost and heat of fusion [6, 13]. Both Kenisarin [13] and Zalba et al. [1] gave extensive lists of salts and eutectics with thermal properties. The toxicity of decomposition products of salts was been highlighted [13] as it was for their use as sensible storage. Review papers consistently discuss the severe limitation of low thermal conductivity and some discuss heat exchanger augmentation methods [8].

Metals have received some attention as Phase Change Materials though the realities of encapsulation and operation have not yet been thoroughly explored. An extensive list of possible eutectics involving Aluminium, Copper, Lead, Magnesium, Silicon and Zinc was given by Liu et al. in 2012 [8]. Another list of suggested alloys is available in Kenisarin's extensive review [13]. Al-Mg-Zn alloy has been proposed as a latent heat storage material by Sun et al. [14] in 2007 though the miscibility of the alloy with its encapsulating material was not identified, hampering long term stability. In 2012 Khare et al. [3] investigated a range of metals using a commercial materials selection program. The authors concluded that eutectics of $\mathrm{Al}, \mathrm{Mg}, \mathrm{Si}$ and $\mathrm{Zn}$ were useful for high temperature storage. An alloy of Al and Si was discussed in 2013 as a potential candidate and its performance with a heat transfer fluid of $\mathrm{NaK}$ was analysed [15]. In space heating some low melting temperature metals were suggested by Tatsidjodoung et al. [9] though their price was given as a critical detriment.

In [16] random growth algorithms were used to replicate the complex geometry of iron-copper and aluminium-tin MGAs. Cross sections of the generated threedimensional models were compared to two-dimensional microscopic images of samples to ensure a representative description of the geometry. Lattice Monte Carlo analyses were conducted in order to calculate the effective thermal conductivity of these MGAs. The study predicts high thermal conductivities $(>100 \mathrm{~W} / \mathrm{m} . \mathrm{K})$ that increase for higher volume fractions of copper (iron-copper MGA) and aluminium (aluminium-tin MGA).

The current study takes this analysis a step ahead by using micro-computed tomography imaging to obtain three-dimensional geometry data of these MGAs. This data is then converted into calculation models for numerical analysis. The result is precise models that guarantee an accurate geometric description of these composites. Lattice Monte Carlo (LMC) simulations are conducted that allow the determination of the effective thermal conductivity, diffusivity, and heat capacity of MGAs. The precise knowledge of these important material parameters is essential for their future application. 


\section{Methodology}

In order to capture the complex geometry of MGAs micro-computed tomography scans of iron-copper $(\mathrm{FeCu})$ and aluminium-tin (AlSn) alloys were obtained. A relatively fine voxel resolution of 1.09 microns was required for an accurate representation of the interface between the metallic phases. The reconstructed geometries are shown in Fig. 1. For better visualisation the matrix phases are drawn transparent. The iron-copper MGA (see Fig. 1a) contains spherical copper particles that are completely enclosed by the iron matrix. The combined volume fraction of the copper particles is $\phi_{\mathrm{Cu}}=0.35$. No residual porosity was identified and accordingly the iron volume fraction is $\phi_{\mathrm{Fe}}=1-\phi_{\mathrm{Cu}}=0.65$. Figure $1 b$ shows the reconstruction of the aluminium-tin MGA. In contrast to the iron-copper system, the fusible phase (Sn) percolates (is interconnected). However, connections between neighbouring Sn flakes occur predominantly through narrow orifices ( $<10$ microns). Accordingly, leakage of the fusible phase at elevated temperatures is unlikely and has so far not been observed during thermal cycling of the material. The volume fractions and residual porosity $p$ of the SnAl sample are $\phi_{\mathrm{Sn}}=0.33, \phi_{\mathrm{Al}}=0.65$, and $p=0.02$.
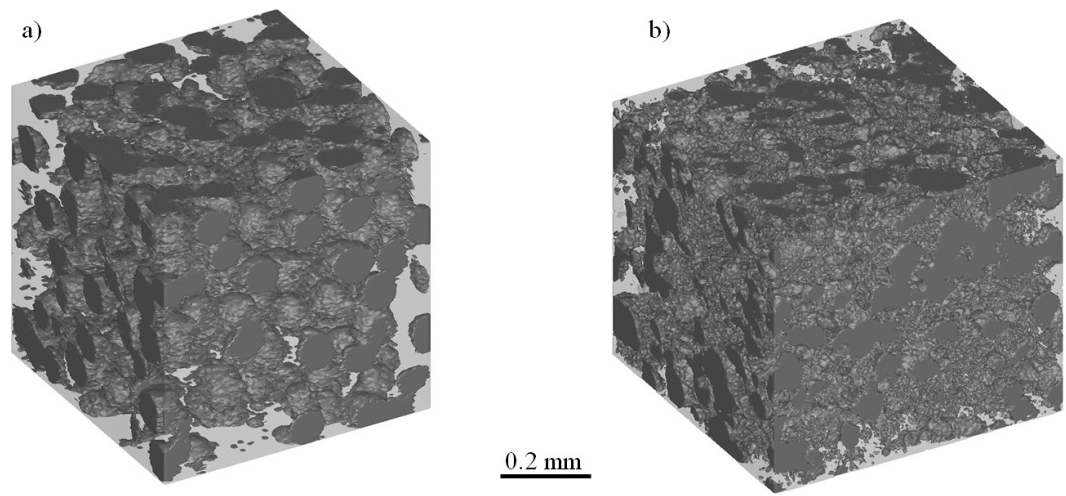

Figure 1: Reconstructed geometries: a) iron (transparent) - copper MGA, b) aluminium (transparent) - tin MGA.

The effective thermal properties of these MGAs were computed using Lattice Monte Carlo analyses. This versatile numerical method allows the computation of the thermal diffusivity tensor of the MGAs. It is based on a large number (in this case $10^{5}$ particles were chosen) of probing particles that diffuse in a Lattice model representing the MGA geometry. In the current study this geometry has been derived from the segmented voxel data obtained by micro-computed tomography imaging. The Einstein diffusion equation then allows the computation of the thermal diffusivity based on the average displacement of the probing particles. A detailed description of the method is available in literature $[17,18]$. The method 
has already been successfully applied for the thermal characterisation of cellular metals [19] and composites [20]. For the computation of the effective thermal MGA properties, the material behaviour of the constituents needs to be defined. These material parameters were obtained from literature and are shown in Table 1.

Table 1: Material properties of the constituents at $293 \mathrm{~K}[21,22]$.

\begin{tabular}{lcccc}
\hline Material & $\begin{array}{c}\text { Conductivity } \\
{[\mathrm{W} / \mathrm{m} . \mathrm{K}]}\end{array}$ & $\begin{array}{c}\text { Density } \\
{\left[\mathrm{kg} / \mathrm{m}^{3}\right]}\end{array}$ & $\begin{array}{c}\text { Heat } \\
\text { capacity } \\
{[\mathrm{J} / \mathrm{kg} . \mathrm{K}]}\end{array}$ & $\begin{array}{c}\text { Thermal } \\
\text { diffusivity } \\
{\left[10^{5} \mathrm{~m}^{2} / \mathrm{s}\right]}\end{array}$ \\
\hline Aluminium & 210 & 2700 & 900 & 8.64 \\
Copper & 398 & 8930 & 385 & 11.6 \\
Iron & 76.2 & 7870 & 440 & 2.20 \\
Tin & 62.2 & 5765 & 256 & 4.21 \\
\hline
\end{tabular}

\section{Results}

First, the effective heat capacity $C_{\text {eff }}$ and density $\rho_{\text {eff }}$ of the MGAs are calculated. The effective density is the volume weighted average of the constituents, i.e.

$$
\rho_{\text {eff }}=\sum_{i=1}^{n} \rho_{i} \Phi_{i}
$$

Using the principle of energy conservation the effective heat capacity can be calculated according to [2]

$$
C_{\mathrm{eff}}=\sum_{i=1}^{n} \psi_{i} C_{p, i}
$$

where $\psi_{i}$ denotes the mass fraction of phase $i$. It should be mentioned here that both the density and heat capacity of the pores in the aluminium-tin MGA are assumed to be zero.

The effective thermal conductivity of the constituents can be estimated using the Maxwell equation, where the subscript 1 represents the percolating matrix (iron / aluminium) and subscript two a segregated particle phase (copper/tin).

$$
k^{*}=k_{1} \frac{2 k_{1} \phi_{1}+k_{2}\left(1+2 \phi_{2}\right)}{k_{2} \phi_{1}+k_{1}\left(2+\phi_{2}\right)}
$$

The superscript * emphasises that unlike equations (1) and (2) the Maxwell equation does not provide an exact solution. In order to obtain precise results for $k_{\text {eff }}$ and $D_{\text {eff }}$ Lattice Monte Carlo simulations were conducted using the geometrical models and material data shown in Fig. 1 and Table 1, respectively. The results for the effective thermal properties are shown in Table 2.

Table 2: Effective thermal properties of MGAs at $293 \mathrm{~K}$.

\begin{tabular}{lccccc}
\hline MGA & $\begin{array}{c}\rho_{\text {eff }} \\
{\left[\mathrm{kg} / \mathrm{m}^{3}\right]}\end{array}$ & $\begin{array}{c}C_{\text {eff }} \\
{[\mathrm{J} / \mathrm{kgK}]}\end{array}$ & $\begin{array}{c}k^{*} \\
{[\mathrm{~W} / \mathrm{mK}]}\end{array}$ & $\begin{array}{c}k_{\text {eff }} \\
{[\mathrm{W} / \mathrm{mK}]}\end{array}$ & $\begin{array}{c}D_{\text {eff }} \\
{\left[10^{5} \mathrm{~m}^{2} / \mathrm{s}\right]}\end{array}$ \\
\hline $\mathrm{FeCu}$ & 8241 & 419 & 135 & 149 & 4.31 \\
$\mathrm{AlSn}$ & 3660 & 565 & 149 & 146 & 7.06 \\
\hline
\end{tabular}


Comparing the results, it becomes apparent that the iron-copper MGA exhibits a distinctly higher density. The simple explanation is the high density of its constituents (see Table 1). The effective heat capacity of the aluminium-tin MGA is $26 \%$ higher indicating a superior ability for specific (per mass unit) sensible heat storage. It is interesting to observe that despite their dissimilar constituents both MGAs have a similar effective thermal conductivity. The results are in good agreement with earlier predictions based on random growth algorithms [16] for the aluminium-tin MGA $(\sim 140 \mathrm{~W} /(\mathrm{m} . \mathrm{K}))$ and the iron-copper MGA $(\sim 157 \mathrm{~W} /(\mathrm{m} . \mathrm{K}))$. The thermal diffusivity of the aluminium-tin MGA is $39 \%$ higher than the corresponding value of the iron-copper system.

The effective thermal diffusivity $k_{\text {eff }}$ listed in Table 2 is averaged over all possible directions. In order to assess thermal anisotropy, a polar plot of the thermal diffusivity is shown in Fig. 2. These values are obtained by evaluating the directional components of the probing particle displacements computed in the LMC analyses. Directional conductivities are shown for three perpendicular (i.e. xy, xz and xy) planes. It should be mentioned here that the orientation of these planes is defined by the positioning of the sample during micro-computed tomography scanning and thus is arbitrary. The plot shows circular graphs with no visible dependence on the selected plane. Accordingly, the effective thermal diffusivity of both materials is isotropic. Corresponding to the average diffusivities shown in Table 2 the radius of the AlSn plot is larger and averages $7.06 \cdot 10^{-5} \mathrm{~m}^{2} / \mathrm{s}$.

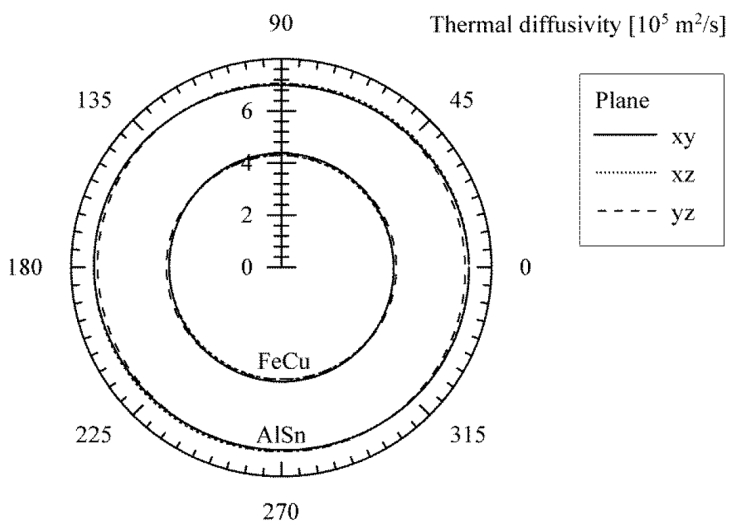

Figure 2: Polar plot of MGA thermal diffusivity.

The storage capacity of MGAs is determined by their ability to absorb thermal energy both in the form of sensible and latent heat. The effective heat capacity $C_{\text {eff }}$ governs specific sensible heat storage. As mentioned above the aluminium-tin MGA has a higher effective heat capacity and thus superior specific sensible heat storage compared to the iron-copper system. However, in the case of thermal capacitors latent energy storage is of greater importance. The advantage of latent over sensible energy storage is temperature stabilization in proximity of the phase transition storage. This allows the delivery of thermal energy (e.g. to a power cycle) at a relatively constant temperature simplifying the design of components such as turbines and simultaneously increasing efficiency. The melting 
temperatures of copper and tin are $1,356 \mathrm{~K}$ and $505 \mathrm{~K}$, respectively. The corresponding heats of fusion are $59.5 \mathrm{~kJ} / \mathrm{kg}$ (tin) and $204.8 \mathrm{~kJ} / \mathrm{kg}$ (copper) $[21,22]$. Considering the mass fractions of the fusible phases the latent heat storages are $30.9 \mathrm{~kJ}(\mathrm{AlSn})$ and $187.6 \mathrm{~kJ}(\mathrm{FeCu})$ per kilogram of MGA. Accordingly, the iron-copper MGA has a six times higher specific storage capacity for latent heat. It should be mentioned here that a large number of additional material systems for MGAs have been identified covering phase transition temperatures from $394 \mathrm{~K}$ (Gallium) to 2,088 K (Palladium). The design, manufacturing and characterisation of these systems will be addressed in our future research.

\section{Conclusions}

The current paper addresses the thermal characterisation of MGA. The effective density, heat capacity, thermal conductivity and thermal diffusivity have been obtained for iron-copper and aluminium-tin MGAs. Both MGAs exhibit similar effective thermal conductivities (146-149 W/m.K). The aluminium-tin system has a higher specific sensible heat storage whereas the iron-copper MGA exhibits superior latent heat storage. Both materials have a high thermal diffusivity (i.e. $4.31 \cdot 10^{-5} \mathrm{~m}^{2} / \mathrm{s}(\mathrm{FeCu}), 7.06 \cdot 10^{-5} \mathrm{~m}^{2} / \mathrm{s}(\mathrm{AlSn})$ ) allowing for rapid storage and discharge of thermal energy which is essential for applications such as concentrated solar power generation. Furthermore, the thermal conductivity and diffusivity of the MGAs are isotropic. The preference of any material system will be governed by the melting temperature of the fusible phase (tin: $505 \mathrm{~K}$, copper: $1,356 \mathrm{~K}$ ) that must closely match the optimum operational temperature of the overall system.

\section{References}

[1] B. Zalba, J. Marìn, L. Cabeza, H. Mehling, Review on thermal energy storage with phase change: materials, heat transfer analysis and applications, Applied Thermal Engineering, 23 (2003) 251-283.

[2] U. Herrmann, D. Kearney, Overview on Thermal Storage Systems, FLABEG, 2002.

[3] S. Khare, M. Dell'Amico, C. Knight, S. McGarry, Selection of materials for high temperature latent heat energy storage, Solar Energy Materials \& Solar Cells, 107 (2012) 20-27.

[4] D. Laing, C. Bahl, T. Bauer, M. Fiss, N. Breidenbach, M. Hempel, HighTemperature Solid Media Thermal Energy Storage for Solar Thermal Power Plants, Proceedings of the IEEE, 100(2) (2012) 516-524.

[5] A. Sharma, V.V. Tyagi, C.R. Chen, D. Uddhi, Review on thermal energy storage with phase change materials and applications, Renewable and Sustainable Energy Reviews, 13 (2009) 318-345.

[6] R. Tamme, Optimised Industrial Process heat and Power Generation with Thermal Energy Storage, ECES, 2010.

[7] M.M. Farid, A.M. Khudhai, S.A.K. Razack, S. Al-Hallaj, A review on phase change energy storage: materials and applications, Energy Conversion and Management, 45 (2004) 1597-1615. 
[8] M. Liu, W. Saman, F. Bruno, Review on storage materials and thermal performance enhancement techniques for high temperature phase change thermal storage systems, Renewable and Sustainable Energy Reviews, 16 (2012) 2118-2132.

[9] P. Tatsidjodoung, N.L. Pierred, L. Luo, A review of potential materials for thermal energy storage in building applications, Renewable and Sustainable Energy Reviews, 18 (2013) 327-349.

[10] S.M. Hasnain, Review on sustainable thermal energy storage technologies, Part I: Heat Storage Materials and Techniques, Energy Conversion and Management, 39(11) (1998) 1127-1138.

[11] M. Sierra, High Efficiency Thermal Storage System for Solar Plants (HELSOLAR), SENER Engineering and System, 2012.

[12] D. Laing, D. Lehmann, M. Fiss, C. Bahl, Test Results of Concrete Thermal Storage for Parabolic Trough Power Plants, Journal of Solar Energy Engineering, 131 (2009) 1-6.

[13] M.M. Kenisarin, High-temperature phase change materials for thermal energy storage, Renewable and Sustainable Energy Reviews, 14 (2010) 955-970.

[14] J.Q. Sun, R.Y. Zhang, Z.P. Liu, G.H. Lu, Thermal reliability test of Al$34 \% \mathrm{Mg}-6 \% \mathrm{Zn}$ alloy as latent heat storage material and corrosion of metal with respect to thermal cycling, Energy Conversion and Management, 48 (2007) 619-624.

[15] J.P. Kotzè, T.W.v. Backström, High Temperature Thermal Energy Storage Utilising Metallic Phase Change Materials and Metallic Heat Transfer Fluids, Journal of Solar Energy Engineering, 135 (2013) 1-6.

[16] A.J. Rawson, E. Kisi, H. Sugo, T. Fiedler, Effective Conductivity of Cu-Fe and Sn-Al Miscibility Gap Alloys, Int. J. Heat Mass. Trans., accepted for publication (14/05/2014).

[17] T. Fiedler, G. E. Murch and I. V. Belova, Solving complex thermal and mass transport problems with the Lattice Monte Carlo method. 7th Pacific Rim International Conference on Advanced Materials and Processing, PRICM7 654-656 (2010) 1476-1481.

[18] T. Fiedler, I.V. Belova, A.J. Rawson and G.E. Murch, Optimized Lattice Monte Carlo for Thermal Analysis of Composites, Comp. Mater. Sci., submitted for publication.

[19] C. Veyhl, T. Fiedler, T. Herzig, A. Öchsner, T. Bernthaler, I. V. Belova and G. E. Murch, Thermal conductivity computations of sintered hollow sphere structures. Metals 2 (2012) 113-121.

[20] T. Fiedler, I. V. Belova, A. Öchsner and G. E. Murch, Lattice Monte Carlo analysis of thermal diffusion in multi-phase materials. Defect and Diffusion Forum 2 (2011) 275-300.

[21] David R. Lide, CRC Handbook of Chemistry and Physics, 80th Edition, CRC Press, Boca Raton, FL (1999).

[22] Alok Nayer, The Metals Databook, McGraw-Hill, New York (1997). 